\title{
CCL21-CCR7 axis in RA: linking inflammation and bone erosion
}

Rheumatoid arthritis (RA) is characterized by joint inflammation and bone erosion mediated by excessive production of proinflammatory mediators. A new study highlights the importance of the chemokine CCL21 and cross-talk between macrophages and $\mathrm{T}$ cells in this destructive process.

In characterizing the pathogenic function of CCL21, the researchers found that the expression of its receptor CCR7 on monocytes was higher in patients with RA than in healthy individuals, and correlated with the patient's 28 -joint disease activity score (DAS28).

In vitro, monocyte chemotaxis, induced by treatment with synovial fluid from patients with RA, was reduced by neutralization of CCL21 or CCR7. Furthermore, treatment with CCL21 promoted the chemotactic activity of monocytes in a CCR7-dependent manner and upregulated the transcription of IL-6 and IL-23 (cytokines involved in Thelper 17 ( $\left.\mathrm{T}_{\mathrm{H}} 17\right)$ cell differentiation).

Given the important function of $\mathrm{T}_{\mathrm{H}} 17$ cells and IL-17 production in RA, the researchers assessed the effects of CCL21 on T cells in vitro. CCL21 treatment promoted IL-17 secretion by peripheral blood mononuclear cells (PBMCs), but not by $\mathrm{T}$ cells alone. Furthermore, CCL21 treatment promoted osteoclastogenesis by PBMCs, which was inhibited by antibodies against IL-6 receptor (IL-6R), IL-23 or IL-17.

These findings together suggest that CCL21 induces polarization of $\mathrm{T}_{\mathrm{H}} 17$ cells in a myeloid celldependent manner, which in turn stimulates osteoclast formation and bone erosion. Notably, adenovirusmediated expression of CCL21 (via intra-articular injection) promoted joint inflammation and

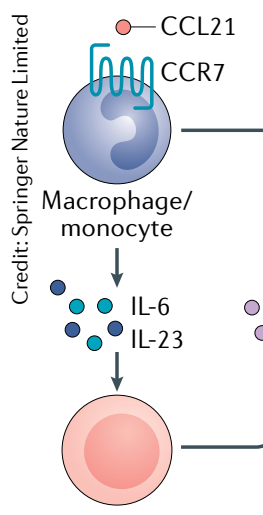

T cell promotes myeloid and $\mathrm{T}$ cell pathogenic activity and is an RA susceptibility gene, CCL21 is ideally positioned to instigate disease and thus makes for a promising novel therapeutic target," explains Katrien Van Raemdonck, first author of the study. "Further research will have to verify whether CCL21-CCR7 activity can be inhibited efficiently and safely for therapeutic purposes."

CCL21 induces polarization of $\mathrm{T}_{\mathrm{H}} 17$ cells in a myeloid cell-dependent manner

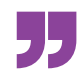

Jessica McHugh

ORIGINAL ARTICLE Van Raemdonck, K. et al. CCL21/CCR7 signaling in macrophages promotes joint inflammation and Th17-mediated osteoclast formation in rheumatoid arthritis. Cell. Mol. Life Sci. https://doi.org/10.1007/s00018-019-03235-w(2019)

\section{Faulty mitochondrial DNA repair promotes inflammation in RA}

In rheumatoid arthritis (RA), T cells age prematurely and have defects in DNA repair mechanisms and a distinct metabolic signature. New research suggests that these characteristics might be linked to each other, and to inflammation, via the mitochondria.

Previous studies had identified low expression of the DNA repair nuclease MRE11A as being linked to the prematurely aged phenotype of RA T cells. In the new study, inducing low MRE11A expression in otherwise healthy $T$ cells produced a phenotype similar to that of $T$ cells from patients with RA, in which mitochondrial function was impaired. Interestingly, the low expression of MRE11A in RA $T$ cells extended to the mitochondria, where it is also present, and was linked to DNA damage and leakage.

"We had to leave our comfort zone and transition from the nucleus to the mitochondria to study how MRE11A contributes to mitochondrial failure in RA T cells," explains corresponding author Cornelia Weyand. "We developed techniques to study how mitochondrial DNA (mtDNA) leaks into the cytoplasm and how leaked mtDNA is recognized by DNA sensors."

The researchers found that leaked mtDNA triggered the inflammasome in T cells with low MRE11A expression, causing the activation of caspase- 1 ,
MRE 11 A knockdown in $T$ cells resulted in increased caspase- 1 activation and synovial tissue inflammation the release of IL- $1 \beta$ and pyroptotic cell death. In a humanized model of RA in which human synovial tissue was engrafted into NSG mice, MRE11A knockdown in T cells resulted in increased caspase-1 activation and synovial tissue inflammation. By contrast, MRE11A overexpression in $T$ cells reduced synovial inflammation in this model.

"Implicating mtDNA repair in the tissue inflammatory response in RA comes as quite a surprise," says

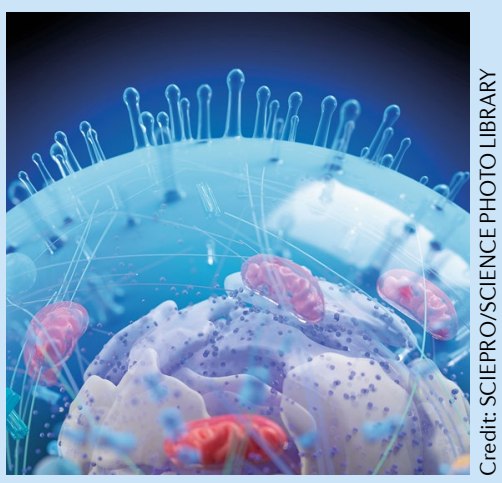

Weyand. "We next want to explore the therapeutic implications of mitochondrial infidelity in RA. Can we prevent the leakage of $m t D N A$ into the cytoplasm? Can we prevent pyroptotic T cell death? Can we repair the damaged DNA within the mitochondria, and will that rescue mitochondrial function?" Future studies will hopefully ascertain whether mtDNA repair is indeed a feasible therapeutic target for RA. Joanna Collison

ORIGINAL ARTICLE Li, Y. et al. The DNA repair nuclease MRE11A functions as a mitochondrial protector and prevents T cell pyroptosis and tissue inflammation. Cell Metab. https://doi.org/10.1016/ j.cmet.2019.06.016 (2019) 